\title{
De nuevo, sobre trabajo y prostitución
}

\author{
Again on work and prostitution
}

\author{
Aurelio Desdentado Bonete* \\ Profesor honorifico de la Universidad Carlos III de Madrid
}

doi: https://doi.org/10.20318/labos.2020.5543

\begin{abstract}
Resumen: $\quad$ Ese artículo aborda el problema de la consideración de la prostitución como un trabajo que puede dar lugar a un contrato laboral. Comienza con una referencia a la dificultad de conocer la prostitución como hecho social y expone las dificultades existentes para el encaje de esa actividad dentro de las características generales que delimitan el supuesto de aplicación de las normas laborales. Continúa con una exposición breve del estado de la cuestión en la doctrina judicial y termina con una referencia a las posibles alternativas.

Palabras clave: contrato de trabajo, prostitución, autonomía privada, voluntariedad, dependencia. imposición legal, moral, orden público.

Abstract: $\quad$ This article addresses the issue of considering prostitution as a job leading to an employment contract. It begins with a reference to the difficulty of recognising prostitution as a social reality and exposes the problems of fitting this activity within the frame of labour law. The article goes on to briefly present the state of the matter in judicial doctrine and closes with a reference to possible alternatives.

Keywords: employment contract, prostitution, private autonomy, voluntary, dependency, legal imposition, morality, public order.
\end{abstract}

\section{La relación entre trabajo y prostitución como problema}

\section{a) Un hecho social complejo, un conocimiento bastante limitado}

Continúo aquí algunos análisis y conclusiones que ya he expuesto en otros trabajos en curso de publicación cuando se redacta éste ${ }^{1}$. Esta persistencia en un tema que tiene su interés, pero también su dificultad, solo puede excusarse por la amable invitación de los profesores Mercader y de la Puebla; una invitación que, dada su autoridad, no he tenido la prudencia de resistir. Ello me obliga ahora a la dura tarea de volver sobre lo mismo, aunque sin perder la esperanza de poder aportar algo nuevo.

adesdent@der-pr.uc3m.es

1 "De Mesalina a OTRAS: la prostitución como trabajo ante los tribunales del orden social", Revista de Jurisprudencia, El Derecho, no 2, marzo 2020 y "Prostitución y contrato de trabajo. Una crítica y una propuesta", pendiente de publicación en el libro colectivo "Los derechos de las mujeres y sus límites" (Cátedra de Estudios Iberoamericanos Jesús Polanco / Universidad Autónoma de Madrid). 
Comenzaré con un reconocimiento de las limitaciones. Creo que no conocemos bien la prostitución como fenómeno social ${ }^{2}$ y ello es un obstáculo importante a la hora de abordar correctamente los problemas y de encontrar soluciones. Así que, con el necesario relativismo y el absoluto respeto a las opiniones contrarias, me limitaré a una exposición de algunos datos y reflexiones que puedan contribuir al necesario debate sobre una situación que no es satisfactoria y que no parece tener una solución clara.

Las dificultades de conocimiento surgen lógicamente porque estamos situados en un ámbito de alegalidad / ilegalidad, en el que se ignoran, alteran o borran los hechos provocando lo que a veces se designa como "invisibilidad" en un entorno difícil y complejo.

Con todo, es útil comenzar con algunas aproximaciones al fenómeno. Desde una perspectiva histórica, se ha señalado que la regulación de la prostitución tuvo en España desde mediados del XIX una orientación reglamentista, con un intento abolicionista en 1935 que quedó sin efecto con la guerra civil ${ }^{3}$. Después, en el primer franquismo, la situación resulta más confusa ${ }^{4}$. A partir de 1956 se impone un abolicionismo de corte tradicional ${ }^{5}$ que, con las lógicas correcciones derivadas del cambio político, se mantendrá, al menos formalmente, hasta que el Código Penal de 1995 incluya una regulación claramente orientada a la protección de la libertad sexual, que, sin embargo, será objeto de algunas restricciones en las reformas posteriores.

Esta es, en muy apretada síntesis, la evolución de nuestro ordenamiento. Pero hay una serie de factores, como el crecimiento del número de personas dedicadas a esa actividad, la conexión con la emigración ilegal y la trata, la mayor percepción social del coste de determinadas externalidades y las reivindicaciones de igualdad desde una perspectiva de género, que han producido en los últimos tiempos una mayor preocupación social.

En esta línea se inscribe el informe sobre la situación actual de la prostitución en nuestro país que se hizo público en mayo de 2007 por la Comisión Mixta de los Derechos de la Mujer y de la Igualdad de Oportunidades de las Cortes. Es, sin duda, un documento, polémico, pero de gran interés ${ }^{6} \mathrm{y}$ con una influencia destacable, que en buena medida persiste. Conviene comenzar, por tanto, con una referencia a él.

El diagnóstico del informe ponía de relieve: $1^{\circ}$ ) la existencia de un volumen de negocio de "dieciocho mil millones de euros al ańo" ${ }^{7}$ con un importante gasto en publicidad ${ }^{8} ; 2^{\circ}$ ) la estimación de unas 400.000 personas dedicadas a esa actividad, cifra integrada fundamentalmente por mujeres; la mayoría "con graves problemas económicos", en muchos casos "en situaciones degradantes", con

${ }^{2}$ C. MARTINEZ MORENO en "La prostitución desde la perspectiva del Derecho del Trabajo" (de próxima publicación en Tirant lo Blanch) destaca que "es difícil saber cuántas personas están ejerciendo en cada momento la prostitución, cuántas realizan su actividad de manera voluntaria, dependiente o independiente" y "en qué tipo de establecimiento". En el mismo sentido E. BOZA MORENO ("La prostitución como trabajo",Tirant lo Blanch, Valencia, 2018, págs.20-24).

${ }^{3}$ J-L GUEREÑA, “La prostitución en la España contemporánea”, Marcial Pons,2003, pág. 451.

${ }^{4}$ Vid. L. GARRIDO GUZMÁN, "La prostitución: estudio jurídico y criminológico”, EDERSA,Madrid, 1992, págs. 156- 160, en una valoración centrada en las normas que contrasta con la práctica social de tolerancia que recoge GUERENA para este periodo.

${ }^{5}$ Con el Decreto - Ley de 3 de marzo de 1956 y luego con la ratificación en 1962 del Convenio de 21 de marzo de 1950, sobre represión de la trata de personas y la explotación de la prostitución ajena.

${ }^{6}$ El informe se publicó en el Boletín Oficial de las Cortes (VIII Legislatura) no 379, 24.5. 2007. Consta de un total de 224 páginas, de las cuales 25 corresponden al informe de la ponencia aprobado por la Comisión y el resto a las intervenciones de los comparecientes. El informe puede verse como un texto político, sin duda significativo, aunque, desde luego, discutible tanto en sus estimaciones como en sus conclusiones. Las aportaciones de los comparecientes tienen especial interés, porque, aparte del valor de sus contribuciones, recogen análisis, opiniones y propuestas que reflejan,desde una perspectiva realmente plural, posiciones de signo diverso por parte de personas cualificadas del ámbito académico o profesional, sindicatos, organizaciones de trabajadores sexuales incluido el sector mal conocido de la prostitución masculina -, asociaciones asistenciales, religiosas y feministas, entidades públicas y representaciones de los cuerpos de seguridad del Estado.

${ }^{7}$ Con una ganancia media anual estimada para los empresarios de $45.000 €$ por persona dedicada a la prostitución (pág. 20).

${ }^{8}$ Con un ingreso estimado de 5 millones de euros por publicidad relacionada con la prostitución para el periódico de mayor tirada (pág. 17). 
de privación de libertad y un fuerte grado de dependencia de organizaciones que las explotan con vulneración de los derechos humanos ${ }^{9} ; 3^{\circ}$ ) la presencia de un alto componente diferencial de género, ya que la mayoría de las personas que practican la prostitución son mujeres, mientras que el 99, $7 \%$ de los consumidores son varones ${ }^{10} ; 4^{\circ}$ ) el alto porcentaje de personas extranjeras que conecta el ejercicio de la prostitución con la emigración ilegal, el tráfico y la trata ${ }^{11} ; 5^{\circ}$ ) la estimación, en términos de demanda, de que "en nuestro país hay 15 millones de varones potenciales clientes" de la prostitución y que el $6 \%$ de la población española es consumidora habitual de prostitución ${ }^{12}$ y $\left.6^{\circ}\right)$ una destacada presencia en el sector de las mafias dedicadas a la trata de personas ${ }^{13}$.

El informe tiene una orientación abolicionista en sus análisis y en sus propuestas. Así, se afirma, por ejemplo, que: $1^{\circ}$ ) la regulación incrementa tanto la prostitución como la trata ${ }^{14} ; 2^{\circ}$ ) el considerar la prostitución como un trabajo " entra en colisión con nuestra normativa en materia de derechos laborales" ${ }^{15} ; 3^{\circ}$ ) a efectos de "la intervención del Estado, nada aporta la distinción entre prostitución libre o forzada", distinción que "olvida que el ejercicio de la prostitución atenta contra la dignidad de las personas que la ejercen" ${ }^{16}$ y $4^{\circ}$ ) la prostitución no supone un ejercicio de la libertad sexual, porque "la sexualidad requiere una situación de igualdad y voluntariedad", en la que "no puede caber" una "relación comercial que constituye en sí misma una situación abusiva, de poder" ${ }^{17}$.

El informe propugna un cuadro muy completo de medidas en orden a erradicar la explotación sexual y la trata de personas, establecer una protección social eficiente y permitir la reinserción laboral en el marco de un plan integral. No podemos examinar aquí esas medidas, aunque haremos alguna referencia a las que se relacionan con la posibilidad de un contrato de trabajo para el ejercicio de la prostitución, una alternativa contra la que el informe se pronuncia tanto de forma general como específica en los términos ya mencionados. Hay que destacar que una de las medidas propuestas era la ampliación del ámbito de ilicitud penal, que partiendo de la existencia de una penalización completa del proxenetismo, proponía "la recuperación de otras figuras desaparecidas como la tercería locativa"18.

Existen lógicamente divergencias con las tesis abolicionistas del informe. Pero también hay discrepancia con las estimaciones. Así, se ha señalado que la cifra de 400.000 mujeres dedicadas a la prostitución en el conjunto del país es excesiva ${ }^{19}$ y que lo mismo ocurre con el cálculo del porcentaje de clientes, pues la referencia a 15 millones clientes "potenciales" solo registraría un grupo de edad, mientras que la mención de un $6 \%$ de la población española como consumidores "habituales" no parece tener un fundamento estadístico claro ${ }^{20}$. En relación con el volumen de negocio, hay que tener en cuenta las estimaciones posteriores del INE sobre la participación en el PIB de las actividades ilegales ${ }^{21}$.

\footnotetext{
${ }^{9}$ Págs. 18, 20 y 22. Aunque también se hace referencia a la existencia de "supuestas ventajas", como "la flexibilidad horaria y de dedicación”, así como la percepción de ingresos "mucho más elevados de lo que cobran mujeres con preparación cultural y laboral igual" (pág. 20).

${ }^{10}$ Págs. 17 y 18. La prostitución masculina tendría también una demanda masculina. Sobre la prostitución masculina puede consultarse la comparecencia del representante de la Fundación Triángulo (págs.71 a 77).

${ }^{11}$ Págs. 18, 20 y 21.

${ }^{12}$ Pág. 20.

${ }^{13}$ Págs. 17, 20 y 22.

${ }^{14}$ Pág. 21.

${ }^{15}$ Págs. 19 y 22.

${ }^{16}$ Pág. 18.

${ }^{17}$ Pág. 19.

${ }^{18}$ Pág. 25.

${ }^{19}$ E. SANCHIS, “La ponencia sobre la prostitución en España”, Claves de Razón Práctica, no 187/ 2008 (págs. 18 y 20). Se refiere a un abanico de estimaciones que van de 100.000 a 500.000, pasando por 300.000 y 400.000. Se considera más razonable la estimación inferior realizada por EDIS.

${ }^{20}$ E. SANCHIS,"La ponencia ...” cit., pág. 20.

${ }^{21}$ BOZA MOORENO, op.cit., págs. 20 y 21. Vid. Instituto Nacional de Estadística, “Contabilidad Nacional de España. Nueva base 2010” en https://www.ine.es/prensa/np862.pdf. El INE advierte que los datos tienen carácter excepcional y solo a efectos in-
} 
Antes y después del informe de la Comisión Mixta fueron apareciendo otros estudios en algunas Comunidades Autónomas, como Cataluña, Andalucía, Castilla y León ${ }^{22}$ y Valencia. En esta última Comunidad se publicó un estudio en 2007, que contiene una estimación muy completa de la prostitución en ese ámbito ${ }^{23}$ y que fija una cifra de 7.560 mujeres (500 en la calle, 5.000 en clubes de carretera y urbanos ${ }^{24}, 1.700$ en pisos, tanto "autogestionados" como "empresariales", y 360 en la denominada prostitución de lujo). Revisada al alza la cifra final para corregir desviaciones de cómputo, se concluye que el número total se podría fijar en torno a 10.000 mujeres para la Comunidad Valenciana, lo que representaría un $10 \%$ sobre la cifra que se acepta para el conjunto nacional.

\section{b) Prostitución forzada y prostitución voluntaria}

Otro punto objeto de debate se refiere al carácter forzado o voluntario de la prostitución. Desde algunas posiciones abolicionistas se ha venido manteniendo que toda prostitución es forzada; afirmación que se justifica tanto por razones de principio ( la prostitución es algo que nadie puede elegir libremente), como en ocasiones aportando altos porcentajes de involuntariedad ( hasta el $95 \%$ en algunos casos ), que vendrían a concluir que el ámbito de la prostitución libre es tan extraordinariamente limitado que no justifica su reconocimiento laboral ${ }^{25}$.

Pero también aquí hay discrepancias ${ }^{26}$, que llevan a la necesidad de hacer distinciones. En primer lugar, es preciso aclarar que el condicionamiento que, en mayor o menor intensidad, experimentan todos los que tienen que vivir de un trabajo no equivale, aunque pueda ser muy apremiante, a una determinación forzosa al mismo mediante violencia o intimidación ${ }^{27}$. Y, en segundo lugar, hay que distinguir también entre una prostitución forzada, más o menos amplia, que es la que opera

formativos. El conjunto de actividades ilegales para 2010 se fija en un 0,87 del PIB; la prostitución representa un 0,35\%, por debajo del tráfico de drogas con un $0,50 \%$.

${ }^{22}$ Un panorama general de estos estudios puede consultarse en F. REY MARTÍNEZ, R. MATA MARTÍN y N. SERRANO ARGÜELLO, "Prostitución y Derecho", Aranzadi, Elcano, 2004, págs. 51- 60.

${ }^{23}$ VV. AA. (I.SERRA, coord.), "La prostitución femenina en la Comunidad Valenciana”, Institut d' Estudis de la Dona, Universitat de Valencia, http://www.inclusio.gva.es/documents/610706/162187124/Prostitucion+femenina+CV/b2270ceb-9918-4deb8488-124cfd671106, en especial y respecto a la cuantificación, págs. 79-108.

${ }^{24}$ Con un claro predominio de los clubes de carretera sobre los urbanos. Una clasificación más general de las modalidades de ejercicio en L. GARRIDO GUZMÁN, op.cit. págs. 70 a 80.

${ }^{25}$ El informe de la Comisión Mixta señala,como hemos visto, que "a efectos de la intervención del Estado nada aporta la distinción entre prostitución libre y forzada”, lo que se justifica con citas del Convenio de Lake Success, del entonces art. 188 del Código Penal y de la resolución del Parlamento Europeo de 2 de febrero de 2006. Pero el que puedan penarse también determinados supuestos de prostitución no forzosa o que se recomiende "luchar contra la idea de que la prostitución es equiparable a un trabajo" no equivale a desconocer las diferencias existentes entre las dos formas de prostitución, que tienen un tratamiento distinto en nuestra legislación penal. Además, el Convenio de Lake Success no tiene aplicación directa, sino a través de las normas penales españolas (STS3a 22.4. 2010, r. 506/07) y tampoco la tiene la resolución del Parlamento Europeo mencionada, que es una declaración política que se limita a realizar recomendaciones.

${ }^{26}$ Para una referencia a estas posiciones y al debate sobre el controvertido porcentaje del $95 \%$ de prostitución forzada, vid. M. LLOBET ANGLÍ, "¿Prostitución?: ni sí, ni no, sino todo lo contrario. Sesgos empíricos,contradicciones de lege data y desaciertos de lege ferenda” (Revista Electrónica de Derecho Penal y Criminología, no 19-19/2017). Tienen interés también,en el Informe de la Comisión Mixta,las divergencias en este punto entre la representante del colectivo Hetaira, Sra. Garaizabal (págs. 40 y 41 ), y la Sra. Fontecha, Secretaria de Igualdad de UGT (págs. 98 y 99). En "Prostitución voluntaria o forzada. Una cuestión a debate" (Papers,96//3, 2011) E. SANCHIS ha abordado el tema a partir de una encuesta realizada a 23 personas, extranjeras y nacionales dedicadas a la prostitución en la Comunidad Valenciana.

${ }^{27}$ Vid. A. MARTÍN VALVERDE, F. RODRÍGUEZ SAÑUDO y J.GARCIA MURCÍA (“Derecho del Trabajo”, Tecnos, Madrid, 2019,págs. 38 y 39), que distinguen entre la compulsión económica al trabajo y el trabajo forzoso: en todas las sociedades históricas la gran mayoría de las personas han tenido que trabajar para vivir, pero "cuando se habla de trabajo libre y trabajo forzoso no nos estamos refiriendo a dicha compulsión", pues solo "existe trabajo forzoso cuando existe imposición del trabajo por parte de otra persona y trabajo libre cuando esta imposición externa no se da". En sentido similar, M. ALONSO OLEA, "Introducción al Derecho del Trabajo" (Civitas, Madrid, 2002, págs.75-78). 
normalmente en el ámbito delictivo de la trata, aunque no solo en éste, y que quedaría por definición fuera del ámbito laboral, y otra voluntaria, en la que sí tiene sentido discutir sobre su eventual carácter laboral cuando se realiza por cuenta ajena.

\section{c) Pero, ¿se trata de un trabajo?}

Hecha esta distinción básica, es conveniente también señalar que la controversia sobre la posibilidad de una relación laboral para el ejercicio de la prostitución se enfrenta con dificultades importantes. Vamos a examinar brevemente algunas de ellas.

En primer lugar, hay que examinar si la prostitución puede definirse como un trabajo, pues el art. 1 ET nos dice que el ordenamiento laboral solo se aplica a los trabajadores, es decir, a quienes realizan voluntariamente un trabajo en determinadas condiciones de ajenidad, dependencia y retribución. No se trata solo de una cuestión teórica, ya que en el informe de la Comisión Mixta se sostiene una posición negativa a la consideración de la prostitución como un trabajo y en las intervenciones de los comparecientes hay también algunos pronunciamientos en este sentido, que invocan consideraciones de orden moral, dignidad y respeto a las mujeres, aparte de hacer referencia a la efectiva garantía de los derechos fundamentales y al rechazo de condiciones degradantes de explotación. Hay también opiniones discrepantes ${ }^{28}$.

Son, unas y otras, respetables, pero plantean un problema que aquí debe abordarse desde la doble perspectiva de la realidad y de la incidencia sobre ella de las consideraciones legales y morales, distinguiendo, por tanto, entre lo que es un trabajo en sí y la calificación jurídica o moral que pueda aplicársele.

A estos efectos, el trabajo debe considerarse ahora en una vertiente más empírica como una actividad humana dirigida a la producción de bienes y servicios mediante un esfuerzo personal. Esa actividad, que puede ser por cuenta propia o ajena, tiene un carácter instrumental, ya que, como se ha señalado, "la característica esencial del trabajo productivo está en que la persona que trabaja pone su esfuerzo teniendo como finalidad la obtención a través de éste de los medios materiales (....) que necesita para subsistir"; una finalidad que "en todo tiempo ha sido y que, desde luego, hoy sigue siendo la motivación dominante de quienes trabajan", pues, en cita de Schopenhauer, "quien hace profesión de una cosa, ama más el beneficio que de ella obtiene que dicha profesión” ${ }^{29}$. Este es el trabajo en sí que es independiente de su consideración como una actividad que puede ser socialmente productiva, improductiva o antiso$\mathrm{cial}^{30}$. En este último caso estaremos ante un trabajo ilícito o delictivo, pero ante un trabajo.

Podríamos recordar aquí, a efectos de distinguir estos dos planos, la doctrina del Tribunal de Justicia europeo, que ha reconocido que la prostitución es una actividad económica en la medida en que "constituye una prestación de servicios remunerada", sin perjuicio de precisar también, en relación con la alegada inmoralidad de esa actividad, que no le corresponde al Tribunal "sustituir por la suya la apreciación de los legisladores de los Estados miembros en los que una actividad supuestamente inmoral se practica legalmente" ${ }^{31}$, aunque tampoco la de otros en los que está prohibida en razón de esa inmoralidad o de consideraciones de orden público.

${ }^{28}$ Vid., por ejemplo, las intervenciones de las Sras. Garaizabal, Juliano y Falcón (págs. 39- 52), de la Sra. Fontecha (UGT, págs. 98-102) y de las representaciones de CC.OO (págs. 33-35 y 65 - 68).

${ }^{29}$ ALONSO OLEA, "Introducción al Derecho del Trabajo", cit., pág. 60.

${ }^{30}$ ALONSO OLEA, op.cit.pág. 62.

${ }^{31}$ STJCE 20.11. 2001 (C 268/99), con criterio reiterado por las SSTJUE 16.12. 2010 (C- 137/09) 1.10.2015 (C 341/14) y 8.5.2019 (C 230 /18). Sobre esta doctrina, vid. A. GUAMÁN HERNÁNDEZ, "La prostitución como actividad económica, la incidencia de la doctrina del Tribunal de Justicia de las Comunidades Europeas en la cuestión" en VV. AA., "Prostitución y trata", Tirant lo Blanch, 2007, págs. 255- 291. 
El Derecho puede, por tanto, rechazar ese trabajo por razones morales o de otra índole; un rechazo que puede también operar con mayor intensidad a través de las normas penales con sanciones de este carácter o, más moderadamente, a través de su exclusión por las normas civiles, laborales o administrativas. Pero esto nos lleva a otras cuestiones.

\section{d) ¿Qué tipo de trabajo?}

Se afirma a veces que la prostitución es un mundo de diversidad y esa diversidad se produce también respecto a la forma en que se ejerce. Hay prostitución por cuenta propia ${ }^{32}$, formas asociativas de ejercicio y prostitución por cuenta ajena, que es la que se realiza a través de un tercero que organiza la actividad, la dirige y la retribuye. ¿Cabe para esta última modalidad un contrato de trabajo?

Hay dificultades importantes y no se refieren solo a las eventuales prohibiciones legales, sino que afectan a la naturaleza de la propia actividad y a los obstáculos para definir una posición empresarial. Así, la prostitución callejera suele presentarse como un típico trabajo por cuenta propia, pero pueden aparecer determinadas "protecciones", que, aparte de su eventual calificación penal, podrían confundirse con posiciones empresariales en un sentido económico (explotación del trabajo ajeno) y en la dirección de la actividad (dependencia). En la actividad que se desarrolla en pisos y en clubs de carretera, las posiciones de dependencia deberían ser más claras, pero cabe su distorsión, recurriendo al juego de otras figuras contractuales, con diferentes combinaciones. Por otra parte, la clasificación formal de las modalidades de ejercicio queda perturbada por la presencia de vínculos con la trata o el tráfico de personas y otras formas forzadas de explotación sexual ${ }^{33}$. Así que la prostitución es también un "espejo oscuro", como subraya una imagen literaria.

En las clasificaciones tradicionales de los llamados "soportes de la prostitución" se suele distinguir entre proxenetas, rufianes y clientes ${ }^{34}$, a los que pueden añadirse las personas dedicadas al ejercicio de la llamada tercería locativa, entendida como una actividad que se limita a proporcionar, a cambio de precio, el local donde se practica la prostitución. Dejando aparte a los clientes y a la tercería, se trata de nociones en general desfasadas, pero que reflejan la complejidad de la actividad. El proxeneta se definía tradicionalmente por una función de mediación, aunque hoy asume sin duda un significado más amplio como el supuesto típico de la posición empresarial en el sector; el rufián suele vincularse con la protección de la persona que ejerce la prostitución, viviendo a su costa y manteniendo en ocasiones con ella algún tipo de relación personal. Estas figuras presentan dificultades a la hora de establecer una posición empresarial, pues con frecuencia los vínculos reales se alteran o se ocultan, de forma que el proxenetismo se presenta como mera tercería locativa y el rufián podría asumir en algunos casos posiciones materialmente empresariales, dirigiendo la actividad, controlándola y recaudando los ingresos ${ }^{35}$. La entrada de la trata y el tráfico de personas añade

\footnotetext{
${ }^{32}$ Es la forma de ejercicio con menos problemas de legalidad, aunque en la práctica pueden surgir dificultades importantes de orden administrativo Sobre la prostitución por cuenta propia, vid. G. POYATOS MATA, "La prostitución como trabajo autónomo" Bosch, Barcelona, 2009 y F. V. LÓPEZ I MORA, “Prostitución y estatuto profesional” en VV. AA., "Prostitución y trata”,cit., págs. 172-178, que examina también el trabajo asociado (págs.178-182).

${ }^{33}$ En este sentido, son ilustrativos los informes presentados a la Comisión Mixta por la Policía Nacional (págs. 77-80) y la Guardia Civil (págs. 132-136).

${ }^{34}$ Una exposición muy detallada de los soportes en GARRIDO GUZMÁN, op.cit., págs. 99- 121.

${ }^{35} \mathrm{Vid}$, por ejemplo, la práctica que recoge el estudio sobre la prostitución en Castilla y León en relación con los establecimientos de carretera: el propietario del hotel o club cobra una tarifa diaria por hospedaje y se compromete a dar al proxeneta el $50 \%$ del dinero generado por las consumiciones y el $30 \%$ de los servicios sexuales; él se queda con otro $30 \%$ y el $40 \%$ restante será para la prostituta. El proxeneta deja a las mujeres en el hotel y no regresa hasta pasados 21 días, fecha en la que retira a las mujeres y hace cuenta con el propietario (REY MARTINEZ y otros, op.cit, pág. 59).
} 
más complejidad, pues el traficante puede ocultarse detrás del proxeneta en una doble posición empresarial ${ }^{36}$.

\section{e) Regular, abolir, prohibir}

¿Hay un margen de licitud para este trabajo cuando se realiza por cuenta ajena? Esta pregunta nos lleva a examinar el sistema de regulación de la prostitución en el que deba encuadrarse el ordenamiento de que se trate, pues de ello depende que haya o no un espacio para un trabajo legal. En este punto se han definido tres modelos. El primero el reglamentismo, que reconoce el ejercicio de la prostitución, pero que la somete a diversos controles de orden público, administrativo o sanitario ${ }^{37}$. El abolicionismo reacciona contra las regulaciones y los controles, que considera degradantes para sostener que hay que erradicar la prostitución, excluyendo su legalización y penalizando a quienes la explotan, pero no a quienes la ejercen. Si la penalización se extiende al cliente, estamos en una versión radical de este modelo. Si alcanza a quien ejerce la prostitución, se trata ya de otro modelo: el prohibicionismo ${ }^{38}$. Con este y con un abolicionismo estricto y coherente no habría espacio para un ejercicio legal de la prostitución.

\section{f) ¿Es viable un contrato de trabajo para el ejercicio de la prostitución?}

Pero los modelos no suelen darse en estado puro. Como hemos visto, en España desde 1956 se adoptó el abolicionismo, que se reforzó con la ratificación del Convenio de Lake Success en 1962. Pronto se impuso, sin embargo, en la práctica una amplia tolerancia que enlazó con una regulación más moderada como consecuencia de la transición política. Con el Código Penal de 1995 parecía que íbamos hacia un reglamentismo liberal, pero no se pasó de la alegalidad. A partir de la reforma de la Ley Orgánica 11/2003, el ámbito delictivo volvió a ampliarse, pero de una forma problemática que dio lugar a una doctrina jurisprudencial moderadora que permitía un margen de licitud ${ }^{39}$. En lo esencial, esta situación parece haberse mantenido con la reforma 2015 en la nueva redacción del art. 187 del Código Penal que no penaliza la explotación de la prostitución voluntaria de adultos cuando no sea abusiva en el sentido que precisan los supuestos de los apartados a) y b) del párrafo $2^{\circ}$ del no 1 de ese artículo. $187^{40}$.

El Derecho Penal no agota la respuesta al problema. Están también los límites que para la autonomía privada establece el art. 1255 CC. Pero, como he señalado en otro lugar, las leyes, aparte

\footnotetext{
${ }^{36}$ Un ejemplo en la STS2a 13.11. 2019 (r. 10121/19) en un caso de prostitución coactiva y trata.

${ }^{37} \mathrm{El}$ reglamentismo ha tenido históricamente una versión clásica muy poco respetuosa con las personas dedicadas a la prostitución y que motivó la reacción del primer abolicionismo de Josephine Butler (J.R. WALKOWITZ, "Sexualidades peligrosas" en VV. AA., "Historia de las Mujeres (IV). El siglo XIX”, págs. 397- 406). Hay, sin embargo, un reglamentismo contemporáneo de carácter más liberal.

${ }^{38}$ Un panorama de estos sistemas en P. DE LORA," ¿Hacernos los suecos? La prostitución y los límites del Estado”, Claves de Razón Práctica, no 187/2008. Desde una perspectiva laboral, vid. S. OLARTE ENCABO, “La prostitución voluntaria: ¿una forma de esclavitud o de ejercicio de libertad personal, de trabajo y de empresa”. La valoración de estos sistemas es variable. El modelo sueco suele presentarse desde perspectivas abolicionistas como un gran logro en la reducción del número de personas dedicadas a la prostitución y de la trata con un coste reducido en términos represivos. Pero los partidarios de la legalización destacan la dificultad de las estimaciones como consecuencia del paso a la clandestinidad y los efectos negativos de ésta.

${ }^{39}$ Una síntesis de esa doctrina en el ATS2a 9.6.2016 (10.092/16), que señala que, en virtud del principio de proporcionalidad, hay que entender que el tipo exige, entre otras circunstancias, que los rendimientos económicos se deriven de "la explotación sexual de una persona que se halle mantenida en ese ejercicio mediante el empleo de violencia, intimidación, engaño o como víctima del abuso de superioridad o de su situación de necesidad o vulnerabilidad".

${ }^{40}$ M. MUÑOZ CONDE ("Derecho Penal. Parte Especial”, Tirant lo Blanch, Valencia, 2019, págs. 239- 241) y E. ORTS BERENGUER en VV. AA., “Derecho Penal. Parte Especial” (Tirant lo Blanch, Valencia, 2019, págs. 256 y 257)
} 
de lo ya dicho sobre las penales, no contienen una exclusión clara. La moral y el orden público son más problemáticos. En el plano de la moral social se registran posiciones diversas, de modo que no es fácil apreciar el consenso necesario para sostener una prohibición cuando se trata de prostitución voluntaria de adultos ${ }^{41}$. Además, la experiencia comparada de los países de nuestro entorno cultural muestra la existencia de soluciones diferentes. Tampoco está en juego el orden público entendido como el conjunto de los principios rectores de la comunidad o como la necesaria reacción ante un riesgo relevante para "un interés fundamental de la sociedad" ${ }^{42}$, sin perjuicio de que lógicamente puedan adoptarse medidas administrativas clásicas para eliminar o corregir externalidades.

Se ha cuestionado también la adecuación del propio contrato de trabajo para regular la práctica de la prostitución por cuenta ajena. Hay, desde luego, en la legislación laboral algunas regulaciones que se ajustan mal o carecen de sentido en esta actividad y que requerirían su eliminación o adaptación en unos términos que no es fácil abordar por vía aplicativa ${ }^{43}$.

La objeción más importante pone de relieve la dificultad o, para algunos, la imposibilidad de admitir el ejercicio de poderes empresariales de dirección, control y disciplina sobre una actividad de carácter tan personal y subrayan que el ejercicio de esos poderes pone en riesgo o vulnera directamente los derechos a la libertad, la intimidad o la integridad personal ${ }^{44}$.

Una posible solución sería, como se ha señalado, una relación laboral especial que permitiera dar respuesta a la singularidad de esta actividad y que, en concreto, garantizara la necesaria "atenuación de la dependencia" ${ }^{45}$ en la línea de otras experiencias comparadas ${ }^{46}$. Pero incluso en el ámbito de la legislación vigente cabe, como han apuntado ya algunas sentencias de instancia, la solución de la laboralidad, aunque haya que adoptar las necesarias cautelas. Una interpretación respetuosa de las normas podría dar respuesta a las exigencias que se derivan del reconocimiento de los derechos fundamentales en cuestión ${ }^{47}$.

Si tampoco se considera posible esta vía, hay que recurrir al art. 9. 2 ET, un precepto que requiere también una interpretación, pues solo garantiza para los contratos nulos - y éste sería el caso

${ }^{41}$ En ocasiones se califica la prostitución como violencia de género, esclavitud o trabajo forzoso. En su significado estrictamente jurídico estas calificaciones no encajan como definición general.No hay violencia si no se impone una acción mediante la fuerza o la intimidación; no hay esclavitud ni trabajo forzado si se trata de un contrato en el que se puede entrar y salir libremente, sin perjuicio de la presión de los condicionamientos económicos que nos obligan a trabajar para vivir. Por otra parte,si hay violencia, imposición forzosa del trabajo o un contrato de esclavitud, no existirá, por supuesto, contrato de trabajo.

${ }^{42}$ STJCE 20.11. 2001, C 268/99)

${ }^{43}$ El problema de la edad mínima laboral se resuelve por la aplicación de las normas penales.Pero hay normas en diversas materias (formación, empleo, clasificación profesional y promoción, ocupación adecuada y extinción del contrato, entre otras) que requerirían exclusiones o adaptaciones.. Sobre estos problemas vid. la completa de exposición de LÓPEZ I MORA (op.cit., págs. 188- 199), que apunta posibles soluciones; también OLARTE ENCABO (op.cit. págs.556- 557) y DE LORA (“ ¿Hacernos los suecos?”,cit., págs. 32y 33).

${ }^{44}$ Está también implicada la dignidad de la persona en una dimensión que se ha relacionado con la cosificación. Remito en este punto a dos artículos recientes de interés: F. ALEMÁN PÁEZ, "El trabajo sexual en la Ley alemana reguladora de la prostitución ("das prostituiertenschutzgesetz"). Bases teóricas y exegéticas de una isonomía crítica” (Derecho de las Relaciones Laborales, $\mathrm{n}^{\circ}$ 7/2018), y M. CUENCA ALARCÓN,”'La libertad sexual y reproductiva e igualdad por razón de género (el supuesto de la aún llamada prostitución)" (Derecho de las Relaciones Laborales no 8/2019). En mi trabajo "Prostitución y contrato de trabajo" he abordado estos problemas a partir de los límites de la imposición legal y de las exigencias del respeto a la autodeterminación personal.

${ }^{45}$ Un examen de las posibles medidas para garantizar la adaptación en R. FERNÁNDEZ, R.TASCÓN, H. ÁLVAREZ y J.G. QUIRÓS, “Sobre la posible regularización laboral de la prostitución: reflexiones para un debate” (Revista de Trabajo y Seguridad Social (CIF),n 24/2007) y LÓPEZ I MORA (op.cit, págs. 182-200). La propuesta de relación laboral especial se ha formulado, entre otros, por FITA ORTEGA (op.cit.,págs. 251-253), CANO GALÁN (op.cit. págs. 628), BOZA MORENO (op.cit.,págs. 67, 68 y 121), C.GALA DURÁN (intervención en la Comisión Mixta de Derechos de la Mujer, cit., pág. 117-120) y el Grupo de Estudios de Política Criminal ("Propuesta de regulación del ejercicio voluntario de la prostitución”, Tirant lo Blanch, Valencia,2010, págs. 24 y 25).

${ }^{46}$ En particular, determinadas medidas previstas en la legislación alemana, como la llamada "prohibición de órdenes" sobre algunos aspectos de la ejecución de la prestación laboral que afectan más directamente a la libertad sexual y la intimidad. Sobre esta legislación, vid. el completo análisis de ALEMÁN PÁEZ en el artículo citado.

${ }^{47}$ Aparte de otras adaptaciones, la limitación de los poderes de dirección empresariales sería posible a la vista de las cláusulas generales de los artículos 4.2.d) y e) en relación con los arts. 19.1 y 20.2 y 3. ET. Por vía interpretativa habría que garantizar la salida de la relación sin necesidad de preaviso. 
- "la remuneración" correspondiente al trabajo que ya se "hubiere prestado". La solución contrasta con la del art. 36.5 de la Ley Orgánica, sobre derechos y libertades de los extranjeros, que va más allá, incluyendo las responsabilidades de Seguridad Social.

Por ello, dada la situación actual, creo que tiene interés detenerse en una opinión discrepante que se puso de relieve como voto particular en una sentencia ya algo antigua, la STSJ Sevilla 4.12. $2003^{48}$. El voto, como veremos, tuvo importancia en la renovación de los argumentos contrarios a la legalización. Era un caso que se presentaba como alterne y en el que se impugnaban las altas de oficio. La sentencia desestimó el recurso de la empresa, confirmando la desestimación de la impugnación del alta en la Seguridad Social por apreciar la laboralidad del alterne. El voto discrepa por entender que hay también práctica de la prostitución, lo que determina la nulidad del contrato. Razona que "no se trata de que califiquemos con cánones éticos la actividad", sino de garantizar "los derechos a la libertad sexual y a la dignidad personal" frente al "ejercicio de los poderes directivos y de organización del empresario", que crean un "evidente peligro de que la prostitución sea forzada y no libre". Sostiene, por tanto, que los contratos deben considerarse nulos, pero sin que "su invalidación afecte a los derechos derivados de la relación de servicios", de forma que procede el alta. Pero, queda también "abierta la posibilidad" de que las afectadas abandonen "la actividad en cualquier momento". Dos puntos hay que resaltar: el contrato es nulo, pero parece que la consecuencia de esa declaración no produce automáticamente la terminación del vínculo, sino que se concreta en reconocer a las afectadas la posibilidad de abandonar en cualquier momento la relación y además los efectos de la prestación de servicios realizada se extienden al alta en la Seguridad Social, aplicando la solución prevista para los extranjeros irregulares.

\section{2. ¿Qué han dicho los tribunales del orden social sobre la prostitución como trabajo?}

\section{a) La posición dominante: exclusión del contrato de trabajo para el ejercicio de la prostitución}

Es este un tema que he abordado de forma más detenida en los artículos ya mencionados, por lo que me limitaré aquí exponer las líneas básicas de la doctrina judicial ${ }^{49}$.

En este sentido creo que la pregunta que abre este apartado tiene en la actualidad una respuesta clara: la prostitución no es en la doctrina judicial dominante un trabajo por cuenta ajena incluido en el ordenamiento laboral. En consecuencia, el contrato laboral para el ejercicio de la prostitución sería nulo, sin perjuicio de la aplicación de las garantías que prevé el art. 9 del Estatuto de los Trabajadores.

Esa es, en síntesis, la respuesta de los órganos judiciales del orden social. Veamos ahora en líneas generales cuáles son los fundamentos de esa conclusión y hasta dónde llega el acuerdo o se produce el desacuerdo.

10) Lo primero que hay que aclarar es que la Sala de lo Social $4^{a}$ del Tribunal Supremo se ha pronunciado en el sentido que se acaba de indicar, pero lo ha hecho de una forma

${ }^{48}$ R 2026. Vid. el comentario de D. DE LA VILLA y DE LA SERNA, "Relaciones laborales de hecho, nulidad del contrato de trabajo y actividades laborales de causa u objeto ilícitos o contrarios a las buenas costumbres" (Revista General de Derecho del Trabajo y de la Seguridad Social no 6/2004), que también pone de relieve la necesidad de generalizar la solución prevista para los emigrantes irregulares.

${ }^{49}$ Remito también para el análisis de la doctrina judicial a los trabajos ya citados de REY MARTÍNEZ y otros, FITA ORTEGA, CANO GALÁN y BOZA MORENO, a los que hay que añadir los de M. GONZÁLEZ DEL RÍO ("El ejercicio de la prostitución y el contrato de trabajo" (Comares, Granada, 2013) y J.F. LOUSADA AROCHENA "El tratamiento legal de la prostitución: ; forma de esclavitud o trabajo sexual?" en "El Derecho ante las formas contemporáneas de esclavitud”, cit., págs. 649- 651). 
indirecta, que es dudoso que podamos calificar como doctrina jurisprudencial en sentido propio. Existen, en primer lugar, las sentencias históricas sobre el alterne de los años 80 del siglo pasado ${ }^{50}$. Pero estas sentencias, aunque aceptaron la laboralidad de ese trabajo, no contemplaron el caso de la prostitución, ni su eventual concurrencia con el alterne. No se dijo que el alterne podía ser laboral, pero la prostitución no, y no se dijo sin duda porque no era necesario, dado el planeamiento de los casos. En realidad, la práctica del alterne era o se fue haciendo más compleja, apareciendo en los nuevos casos relaciones problemáticas con la prostitución, a las que luego se hará referencia.

2o) Hay una sentencia bastante posterior, la STS 27.11. 2004 ${ }^{51}$, dictada en el caso "Mesalina”, que suele considerarse más explícita. Es una resolución de interés, pues resuelve un caso en el que se había denegado la inscripción de una asociación empresarial de la Ley 19/1977 por la conexión de su ámbito con el ejercicio de la prostitución. Pero, en realidad, ese ámbito se definía en función de la actividad de los asociados a la patronal y la actividad quedaba referida a "la tenencia o gestión o ambas, de establecimientos públicos hoteleros destinados a dispensar productos o servicios que tengan como público objetivo terceras personas, ajenas al establecimiento, que ejerzan el alterne y la prostitución por cuenta propia”. Por tanto, a efectos de la inscripción como organización patronal, no se planteaba el problema de una eventual actuación en unas hipotéticas relaciones colectivas de trabajo entre los empresarios asociados y las personas dedicadas a la prostitución, ya que éstas eran solo clientes y además actuaban por cuenta propia. Se confirmó, por tanto, el fallo de la Audiencia Nacional favorable a la inscripción de la asociación empresarial, pero precisando, por una parte, que la acción colectiva de la patronal hacía referencia al personal laboral de los establecimientos ( " camareros, limpiadoras, etc.), incluido "el alterne en su caso, cuando (...) sea laboral", y advirtiendo, por otra parte, que si en "el curso de su actuación futura" pudiera apreciarse un fomento de la prostitución, "habrán de adoptarse las medidas oportunas por quien corresponda". No hay pronunciamiento explícito, porque no es necesario en el caso, aunque se está indicando que cabe el alterne laboral, pero no la prostitución laboral.

$3^{\circ}$ ) El protagonismo en la elaboración de la doctrina judicial ha correspondido, por tanto, a los órganos de instancia y de suplicación, decidiendo normalmente procedimientos de oficio y, más raramente, despidos, lo que no deja de ser significativo. Nos ocuparemos solo de las sentencias de suplicación, que con bastante frecuencia han tenido que entrar en un marco de relaciones muy complejas entre alterne y prostitución; un problema que todavía no tiene una solución uniforme. No puedo ocuparme aquí de este tema pero hay que destacar su interés, ya que, nos ilustra sobre las nuevas formas de ejercicio de la prostitución y además, desde una perspectiva jurídica, pone de relieve un tema doctrinal importante: el de los negocios complejos o coaligados: alterne laboral por cuenta ajena o propia y prostitución también por cuenta ajena o por cuenta propia, combinada en ocasiones con el arrendamiento de habitaciones en el propio establecimiento ${ }^{52}$.

Dejando al margen las dificultades derivadas de las conexiones, las sentencias de suplicación coinciden en la solución del problema principal: no es posible un contrato de trabajo para el ejer-

\footnotetext{
${ }^{50}$ Las sentencias históricas van de la STS 3.3.1981 (RJ 1301) a la STS 4.2.1988 (RJ 571).

${ }^{51}$ R. $18 / 2004$.

52 ¿Qué hacer en estos casos?, ¿hay dos o tres relaciones independientes?, ¿prevalece la principal?, ¿la prostitución desplaza siempre al alterne?. Pueden confrontarse, por ejemplo, las soluciones de las SSTSJ Galicia 16.1.2015 (r. 3656/13) y STSJ Cataluña 2. 10.2008 (r. 943/06).
} 
cicio de la prostitución. Pero, si la conclusión es uniforme, la argumentación se va haciendo más compleja, aunque no contradictoria. En las primeras sentencias, bastante "escasas", como se ha dicho ${ }^{53}$, encontramos la argumentación tradicional que se funda en los límites del contrato por ilicitud del objeto o de la causa (art. 1255 en relación con los arts. 1271 y 1275 del Código Civil). Ahora bien, conforme avanza el nuevo siglo y probablemente como consecuencia del nuevo Código Penal de 1995, los pronunciamientos son más frecuentes y la fundamentación se hace más compleja con la referencia a la vulneración de los derechos fundamentales (la libertad sexual, la integridad y la intimidad) y mediante la incorporación de algunas posiciones del abolicionismo como la referencia a la violencia de género, la esclavitud o el trabajo forzoso ${ }^{54}$. En este sentido, resulta significativa la argumentación de la STSJ Cataluña 15.5.2009 ${ }^{55}$, en la que se señala que "no entra ni puede entrar dentro de las facultades del empresario que regula el art. 20 ET el disponer sobre el uso del cuerpo de la propia persona que trabaja, pues "no es esa persona el objeto del contrato laboral", lo contrario sería "una interpretación aberrante y fraudulenta que intenta asimilar la venta de la fuerza de trabajo" a "la venta del uso del cuerpo de los trabajadores", algo que sería parecido a "la relación de esclavitud". Esta nueva fundamentación se ha ido generalizando, ${ }^{56}$ concurriendo en muchos casos con la tradicional a partir del art. 1255 CC.

40) Es importante destacar también que después del caso Mesalina el Tribunal Supremo no ha abordado el problema de forma directa, aunque en ocasiones, al inadmitir recursos de unificación de doctrina, se incorpora alguna referencia obiter dicta a la exclusión del contrato de trabajo para el ejercicio de la prostitución ${ }^{57}$.

Por ello, si tenemos en cuenta lo dicho sobre las sentencias históricas del alterne, las limitaciones de la decisión del caso Mesalina y las que se derivan de las nuevas resoluciones del Tribunal Supremo - sentencias o autos de inadmisión -, hay que concluir que no existe jurisprudencia sobre el problema.

5o) De ahí la conveniencia de cerrar la exposición de este apartado con una mención de la sentencia de la Sala de lo Social de la Audiencia Nacional de 19 de noviembre de $2018^{58}$, que ha resuelto un nuevo caso famoso, el del sindicato OTRAS. La sentencia, que está pendiente de recurso de casación, tiene interés, porque se pronuncia de nuevo sobre un problema de carácter colectivo: un sindicato de trabajadores del sexo, cuyos estatutos se anulan por considerar que está incluida en su ámbito la prostitución por cuenta ajena. Pero es importante también porque en esta resolución se contiene una completa síntesis de los argumentos, tanto tradicionales como innovadores, que excluyen la laboralidad de la prostitución por cuenta ajena.

${ }^{53}$ REY MARTÍNEZ y otros (págs.195 -213), que examinan también decisiones de los órdenes civil y contencioso-administrativo. Pueden citarse las SSTJS Islas Baleares 9.1.1992 (AS 201), Cataluña 2.1.1997 (AS 340) y 17.9.2003 (r. 3826/03); esta última aceptando la laboralidad del alterne concurrente con la prostitución.

${ }^{54}$ Esta renovación se inicia con el voto particular de la STSJ Sevilla 4.12.2003 (2026/03), que ya se ha examinado y que se funda en los derechos constitucionales a "la libertad sexual y a la dignidad". El paso hacia los argumentos con perspectiva de género puede verse en la STSJ Galicia 10.11. 2004 (r. 3598/04), que acepta los de la sentencia de instancia (SJS Vigo 7.5.2004 (p.803/03). Se aprecia la nulidad de los contratos de trabajo que tengan por objeto el ejercicio de la prostitución, "al ser la explotación de la prostitución por cuenta ajena una forma de violencia de género" y de "esclavitud de las mujeres", aunque también ańade una referencia a la moralidad.

${ }^{55} \mathrm{R} 101 / 08$. La sentencia admite como laboral el alterne concurrente con la prostitución, lo que motiva un voto particular.

${ }^{56}$ Por ejemplo, en las SSTSJ Galicia 16.1.2015 (r. 3656/15) y 12.6.2019 (r. 753/19); también en las del TSJ de Cataluña de 4.7.2013 (r. 3656/12) y 15.11.2013 (4248/13) y en la SSAN 19.11. 2018, p. 258/18). La STSJ Cataluńa 11.11. 2019 (r. 3647/19) adopta también una perspectiva de género, pero se centra en la lesión de los derechos fundamentales a la libertad sexual y la intimidad.

${ }^{57}$ SSTS 29.10.2013 (r. 61/139) y 21.12. 2016 (r. 1778 y 1868/ 15), que citan numerosos autos de inadmisión.

${ }^{58}$ P. 258/18. 


\section{b) Una solución alternativa: la prostitución puede dar lugar a un contrato de trabajo}

Este es, en resumen, el panorama de la doctrina judicial. Ha surgido, sin embargo, una discrepancia cualitativamente importante, que se puso de relieve hace ya algunos años en la sentencia del Juzgado de lo Social no 10 de Barcelona de 18 de febrero de $2015^{59}$. La sentencia, con una argumentación innovadora, decide en un procedimiento de oficio sobre el caso de un establecimiento que se presentaba como centro de masajes. Quedó probada la práctica de la prostitución y la presencia de datos que confirmaban las notas de ajenidad y dependencia, sin que se apreciara ningún elemento de coacción o presión por parte de la empresa o de terceros.

La sentencia reconoce que, aplicando los criterios de la doctrina judicial vigente, el contrato de trabajo debería ser excluido. Pero entiende que hay que reconsiderar esa conclusión. En primer lugar, en atención a las siguientes consideraciones: $1^{\text {a) }}$ la legislación penal vigente ${ }^{60}$ deja un margen de licitud a la explotación de la prostitución de adultos cuando, como en el caso, es voluntaria y no se realiza en condiciones abusivas, y $2^{\mathrm{a}}$ ) no hay en estos casos, en principio, una vulneración de los derechos fundamentales que suelen invocarse por la doctrina judicial para justificar la ilicitud, porque, con cita de la doctrina científica, se razona que un contrato libremente aceptado no puede implicar una violación de la libertad, la intimidad o la dignidad.

Ahora bien, el principio de igualdad visto desde la perspectiva de género y desde el ámbito europeo plantea al juez más problemas. Para abordarlos se examina tanto la STJCE 20.11. 200161, como la resolución del Parlamento Europeo de 26.2.2014, sobre la explotación sexual y la prostitución y su impacto en la igualdad de género. La sentencia del Tribunal de Justicia, considera, como ya hemos visto, que la prostitución ejercida de manera independiente está incluida en la noción de "actividades económicas", lo que puede verse como una forma de normalización. Pero, aparte de que esto no resolvería el problema de la prostitución por cuenta ajena, el magistrado afirma que los problemas se plantean a la vista de la resolución del Parlamento Europeo mencionada, en la que hay una condena muy contundente de la legalización de la prostitución que se reproduce en parte ${ }^{62}$. De todas formas y reconociendo que la resolución no tiene carácter normativo, la sentencia concluye señalando que en la situación actual y hasta que el Estado español " no asuma (...) el modelo nórdico de lucha contra la prostitución", no puede rechazar "el reconocimiento del carácter laboral" de la relación, ya que ello, en la actual "situación de alegalidad", lo que haría sería "agravar enormemente la incuestionable lesión de la dignidad, la libertad y la igualdad que comporta toda relación por cuenta ajena para la inmensa mayoría de las mujeres que la ejercen”.

Pese al destacable esfuerzo argumental con el que ha justificado su innovadora posición, la influencia de esta sentencia en la práctica judicial ha sido limitada. Solo conozco dos resoluciones judiciales de instancia - una de ellas resolviendo un caso de prostitución masculina - que hayan mantenido esta posición ${ }^{63}$.

\footnotetext{
${ }^{59}$ P. $835 / 13$

${ }^{60}$ El art. 188. 1 del Código Penal en la versión de la Ley Orgánica 1/2003. No había entrado en vigor la reforma de 2015.

${ }^{61}$ Vid. supra 1.c).

${ }^{62}$ Veamos algunas de las afirmaciones: $1^{\text {a) }}$ no solo la prostitución forzada, sino también la prostitución en general, representan una forma de esclavitud incompatible con la dignidad de la persona, los derechos fundamentales y la Carta de los Derechos Fundamentales de la Unión Europea, $2^{a}$ ) están además ligadas a la desigualdad de género, $3^{\circ}$ ) la prostitución tiene consecuencias físicas y psicológicas devastadoras, 4a) la legalización del "trabajo sexual", la despenalización de la industria del sexo y la legalización del proxenetismo son contraproducentes y aumentan el riesgo de violencia y el número de víctimas. La sentencia recuerda también que en su punto 29 la resolución del Parlamento considera que el sistema más adecuado para luchar contra el tráfico de mujeres y menores con fines de explotación sexual, mejorando la igualdad de género, es el denominado modelo nórdico, en el que la compra de servicios sexuales constituye delito.

${ }^{63}$ Se trata de la SJS no 33 Barcelona 14.9.2017 (p. 938/16) y de la SJS no 32 Barcelona 10.12. 2018 (p. 149/18 E). La segunda, que trata de la prostitución masculina, ha sido revocada por la STSJ 11.11.2019, una sentencia de indudable interés que ya se ha men-
} 


\section{3. ¿Qué podemos hacer?}

Para terminar quiero subrayar que no estamos solo ante un problema más o menos complejo sobre la interpretación de las normas vigentes. Se trata de una cuestión política en la que surgen además preguntas bastante radicales de orden moral y social.

Por eso he comenzado estas notas con una referencia, quizá demasiado extensa, al informe de la Comisión Mixta de Derechos de la Mujer. Pese a las críticas de que ha sido objeto, creo que fue una iniciativa positiva que permitió un debate que era necesario y que habría que reabrir, partiendo de un análisis completo y neutral de una realidad tan difícil de conocer.

En cualquier caso, los acuerdos no son fáciles. Y no se trata solo de determinar lo que consideramos bueno o conveniente en el ámbito de los planes de vida. Estamos ante un viejo problema de la Filosofía del Derecho: el de la imposición legal de la moral. ¿Hasta dónde pueden llegar las leyes?, ¿qué costes puede tener en términos de libertad y de protección social? Aquí nos encontramos de nuevo con el eterno retorno del debate sobre las relaciones entre la ley y la moral o entre la justicia y la utilidad.

Se ha dicho que la situación legal en nuestro país es "insatisfactoria, contradictoria e hipócrita" ${ }^{64}$. Sin duda es posible calificarla así, dada la distancia entre la realidad y la norma y las dificultades para obtener conclusiones claras en puntos importantes. Pero también podría verse esa ambigüedad como una solución moderada de equilibrio ante unas posiciones que no son fácilmente conciliables.

Hay, por tanto, tres opciones: mantener la situación actual, procurando dentro de lo posible evitar las situaciones de desprotección, adoptar un abolicionismo decidido en la línea del modelo sueco o pasar a una regulación que permita un ámbito de legalidad para la prostitución no forzada de adultos con adaptaciones que tampoco son fáciles.

cionado en la nota 56. En la línea que excluye la laboralidad puede consultarse también la SJS no 24 Madrid 25.7.2017 (p. 389/17), revocada solo en parte por la STSJ Madrid 18.2. 2019 (868/18), que acepta la laboralidad del alterne, pero no de la prostitución.

${ }^{64}$ OLARTE ENCABO op.cit., pág. 552. 